Canadian University Music Review

Revue de musique des universités canadiennes

\title{
Les œuvres de Claude Vivier
}

Numéro 4, 1983

URI : https://id.erudit.org/iderudit/1013896ar

DOI : https://doi.org/10.7202/1013896ar

Aller au sommaire du numéro

Éditeur(s)

Canadian University Music Society / Société de musique des universités canadiennes

ISSN

0710-0353 (imprimé)

2291-2436 (numérique)

Découvrir la revue

Citer ce document

(1983). Les œuvres de Claude Vivier. Canadian University Music Review / Revue de musique des universités canadiennes, (4), 17-21.

https://doi.org/10.7202/1013896ar

(c) Canadian University Music Society / Société de musique des universités canadiennes, 1983
Ce document est protégé par la loi sur le droit d'auteur. L'utilisation des services d'Érudit (y compris la reproduction) est assujettie à sa politique d'utilisation que vous pouvez consulter en ligne.

https://apropos.erudit.org/fr/usagers/politique-dutilisation/ 


\section{LES OEUVRES DE CLAUDE VIVIER}

1968 OJIKAWA

pour soprano, clarinette et percussion

QUATUOR A CORDES

1969

PROLIFERATION (révisé en 1975)

pour ondes martenot, piano et percussion

1970 HIEROPHANIE

pour soprano et petit ensemble

1972

DEVA ET ASURA

pour cordes et bois

DÉSINTÉGRATION

pour deux pianos, deux violons et deux altos

1973 CHANTS

pour deux sopranos, trois mezzo-sopranos et deux contraltos

1974

O! KOSMOS

pour choeur à quatre voix

MUSIK FÜR DAS ENDE pour vingt voix

LETTURA DI DANTE

pour soprano et petit ensemble

JESUS ERBARME DICH

pour choeur à quatre voix

1975

POUR VIOLON ET CLARINETTE

POUR GUITARE

PIANOFORTE

pour piano solo

POUR FLÔTE ET PIANO

Revue de musique des universités canadiennes, $\mathrm{N}^{\circ} 4,1983$ 
POUR VIOLON ET PIANO

POUR VIOLONCELLE ET PIANO

POUR BASSON ET PIANO

HYMNEN AN DIE NACHT

pour soprano et piano

\section{LIEBESGEDICHTE}

pour solistes et petit ensemble

1976

LEARNING

pour quatre violons et percussion

SIDDHARTHA

pour orchestre

NANTI MALAM

ballet en une scène pour sept voix

LES COMMUNIANTES

pour orgue

1977 SHIRAZ

pour piano solo

PARAMIRABO

pour flûte, violon, violoncelle et piano

PULAU DEWATA

pour n'importe quelle combinaison d'instruments

JOURNAL

pour quatre solistes, choeur à quatre voix et percussion

LOVE SONGS

ballet en une scène pour sept voix

version de concert pour sept voix

1978 GREEN

pour flûte, violon, violoncelle et piano

1979 ORION

pour orchestre

GREETING MUSIC

(instrumentation non précisée)

1980

CINQ CHANSONS POUR PERCUSSION

percussion solo

LONELY CHILD

pour soprano et orchestre 


\section{ZIPANGU}

pour orchestre à cordes

L'OPÉRA KOPERNIKUS opéra en deux scènes

1981

PRÉLUDE POUR UN MARCO POLO

pour soprano, contralto, ténor, baryton, basse, six clarinettes, deux percussions et cordes

SAMARKAND

pour piano et quintette à vents

BOUCHARA

pour soprano solo, quintette à cordes, quintette à vents et percussion

\section{A LITTLE JOKE}

pour choeur a capella

WO BIST DU LICHT ?

pour mezzo soprano, vingt cordes, percussion et bande

ET JE REVERRAI CETTE VILLE ÉTRANGE pour alto, violoncelle, contrebasse, trompette et percussion

1982

TROIS AIRS POUR UN OPÉRA IMAGINAIRE pour soprano, cordes, six vents et percussion

CROIS-TU EN L'IMMORTALITÉ DE L'ÂME ? (inachevé) pour trois sopranos, trois altos, trois ténors, deux basses, un baryton, trois synthétiseurs et percussions

\section{Discographie}

PROLIFÉRATION (1968-69)

Jean Laurendeau, ondes martenot

Louis-Philippe Pelletier, piano

Serge Laflamme, percussion

RCI (Radio-Canada International) 358 épuisé

PULAU DEWATA (1971)

McGill Percussion Ensemble

RCI 478 
LETTURA DI DANTE (1974)

Pauline Vaillancourt, soprano

Ensemble de la Société de Musique Contemporaine du Québec direction Serge Garant

RCI 411

POUR FLÛTE ET PIANO (1975)

Lise Daoust, flûte

Suzanne Blondin, piano

RCI 535

On peut se procurer les disques disponibles au prix de $8.98 \$$ (1983) en écrivant à "CBC Enterprises », C.P. 500, Toronto, Ontario, Canada, M5W 1E6

\section{CHANTS (1972)}

sept voix de femmes dirigées par Lorraine Vaillancourt Couplé avec les cinq pièces pour orchestre op. 16 de Schoenberg dans l'arrangement pour deux pianos de Anton Webern

(Lorraine et Jean-Eudes Vaillancourt, pianistes)

" Nocturnales » CCL33-131

On peut se procurer ce disque au prix de $7.95 \$$, plus $8 \%$ de taxe pour les résidents du Québec et 1 \$ pour frais de manutention, en écrivant à la Faculté de Musique, Université de Montréal, C.P. 61-28, Succursale A, Montréal, P.Q., Canada, H3C 3J7

\section{LONELY CHILD (1980)}

Marie-Danièle Parent, soprano

Orchestre Métropolitain du Grand Montréal

direction Serge Garant

PULAU DEWATA (1971)

Douglas Perey, alto

Henry Kucharzyr, piano

Beverley Johnston, percussion

SHIRAZ (1977)

Louis-Philippe Pelletier, piano

CMC-1384

Ce disque reproduit en partie l'enregistrement du concert commémoratif présente à la salle Claude Champagne de Montréal le 2 juin 1983. On peut se le procurer dans tous les centres de 
musique canadienne du Canada (à Montréal, Toronto, Calgary et Vancouver) ou le commander par correspondance (12 \$ canadiens) au Centre de musique canadienne, 430 rue Saint-Pierre, N³00, Montréal, P.Q., Canada, H2Y 2 M5. 